\title{
Improved retinal and visual function following panmacular subthreshold diode micropulse laser for retinitis pigmentosa
}

\author{
Jeffrey K. Luttrull ${ }^{1}$
}

Received: 8 August 2017 / Revised: 9 December 2017 / Accepted: 17 December 2017 / Published online: 16 February 2018

(c) The Author(s) 2018. This article is published with open access

\begin{abstract}
Objectives To examine the effect of subthreshold diode micropulse laser (SDM) on pattern electroretinography (PERG) and visual function in retinitis pigmentosa (RP).

Methods The records of all patients (pts) undergoing SDM in a vitreoretinal subspecialty practice were reviewed. Inclusion criteria included the presence of RP evaluated before and after SDM by PERG. As a secondary outcome measure, the results of automated omnifield resolution perimetry (ORP) were also reviewed.

Results All eyes undergoing SDM for RP were eligible study, including 26 eyes of 15 pts; seven male and eight female, aged 16-69 (avg. 47) years. Retinal function by PERG improved by all indices, with significant improvements in the $24^{\circ}$ field signal latency measures; the $\operatorname{MagD}(\mu \mathrm{V}) / \operatorname{Mag}(\mu \mathrm{V})$ ratio $(P<0.0001)$ and the $\operatorname{MagD}(\mu \mathrm{V})$ amplitude $(P=0.0003)$. ORP significantly improved by all indices $(p=0.02-0.002)$. Average best-corrected chart visual acuities improved from 0.6 to 0.4 $\log$ MAR units $(p=0.02)$. There were no adverse treatment effects.

Conclusions SDM significantly improved chart visual acuity, mesopic logMAR visual acuity perimetry, and retinal function by PERG in RP without adverse treatment effects. Treatment responses indicate a significant capacity for rescue of dysfunctional retina. These results suggest that early and periodic treatment with SDM might slow disease progression and reduce long-term vision loss.
\end{abstract}

\section{Introduction}

Retinitis pigmentosa (RP) is the fourth leading cause of irreversible visual loss in the world [1]. Over 150 different genotypes have been identified which may lead to the clinical picture of RP, presenting diagnostic and therapeutic challenges [2]. Currently, despite great advances in drug, genetic, and immunotherapy, there is no generally effective treatment [3].

Recently, improved retinal and visual function were reported in patients receiving panmacular subthreshold

Electronic supplementary material The online version of this article (https://doi.org/10.1038/s41433-018-0017-3) contains supplementary material, which is available to authorized users.

Jeffrey K. Luttrull

jkluttrull@gmail.com

1 Private practice, 3160 Telegraph Rd, Suite 230, Ventura 93003 CA, USA diode micropulse laser (SDM) as retinal protective therapy for dry AMD and inherited chronic progressive retinopathies (CPRs), including retinitis pigmentosa, Stargardt's disease, and cone degeneration [4]. This report focuses on the electrophysiologic and visual responses to SDM in a larger group of patients with RP.

\section{Methods}

This study adhered to the tenets of the Declaration of Helsinki. Following approval by an investigational review board, the records of all patients undergoing SDM in a private vitreoretinal subspecialty practice were identified and eyes treated for RP identified. Each patient had been evaluated prior to treatment by clinical examination, fundus photography, and spectral-domain optical coherence tomography (OCT). The diagnosis of RP had been previously confirmed in each patient by electroretinography (ERG). All patients exhibited typical clinical features of RP, reported nyctalopia, and circumferential peripheral visual field 
constriction confirmed by prior to conventional automated threshold perimetry. Each eye was evaluated by pattern electroretinography (PERG) 1 week prior, and 1 month after, SDM treatment [5-9]. All, but the initial two vpatients were also evaluated by Omnifield resolution perimetry (ORP) concurrent with PERG testing. Four eyes of two patients reported previously are included in this report [4].

Exclusionary criteria included other obfuscating ocular disease; inability to perform or cooperate with diagnostic testing or treatment; poor signal quality and/or excessive (>3) PERG testing artifacts per study; and failure to followup for postoperative evaluation and testing.

Untreated fellow eyes served as controls.

\section{PERG testing}

PERG was performed using standard protocols of a commercially available and FDA approved system (Diopsys Nova-ERG, Diopsys Corp., Pine Brook, New Jersey) according to the International Society for Clinical Electrophysiology of Vision standards [5]. Both eyes were tested simultaneously and recorded individually, undilated, and refracted for the $60 \mathrm{~cm}$ testing distance. For all visual stimuli, a luminance pattern occupying a $25^{\circ}$ visual field is presented with a luminance reversal rate of $15 \mathrm{~Hz}$.

The PERG "concentric ring" (CR) visual stimulus was employed, presenting alternating (pattern reversal) $\mathrm{CR}$ of contrasting luminance. The $\mathrm{CR}$ stimulus used two sub-classes of stimulus area, with the circle occupying a visual field of either $16^{\circ}$, or $24^{\circ}$, respectively. The stimuli used a mean luminance of $117.6 \mathrm{~cd} / \mathrm{m}^{2}$ with a contrast of $100 \%$.

Patient and equipment preparation and testing were carried out according to Diopsys manufacturer guidelines. Signal acquisition and analysis followed a standard screening protocol [6]. Test indices available for analysis included "Magnitude D $(\operatorname{MagD}(\mu \mathrm{V}))$ ", "Magnitude $(\mu \mathrm{V})$ ", and the "MagD $(\mu \mathrm{V}) / \mathrm{Mag}(\mu \mathrm{V})$ " ratio. $[\operatorname{MagD}(\mu \mathrm{V})]$ is the frequency response of the time-domain averaged signal in microvolts $(\mu \mathrm{V}) . \operatorname{Mag}(\mathrm{D})$ is a measure of signal latency caused by inner retinal and/or ganglion cell dysfunction resulting in both magnitude and phase variability that reduce MagD by phase cancelation. Magnitude $(\mu \mathrm{V})(\mathrm{Mag}$ $(\mu \mathrm{V}))$ measures the frequency response of the total signal in microvolts $(\mu \mathrm{V})$. Mag $(\mu \mathrm{V})$ reflecting the signal strength and electrode impedance of the individual test sessions, as well as a gross measure of inner retina and ganglion function. The $\operatorname{MagD}(\mu \mathrm{V}) / \mathrm{Mag}(\mu \mathrm{V})$ ratio provides a measure of patient response normalized to that particular test's electrical quality, correcting for inter-test variation. In the healthy eye, $\operatorname{MagD}(\mu \mathrm{V})$ should roughly equal $\operatorname{Mag}(\mu \mathrm{V})$. Thus, the closer
$\operatorname{MagD}(\mu \mathrm{V}) / \operatorname{Mag}(\mu \mathrm{V})$ to unity, the more normal retinal function. If testing revealed poor signal quality or excessive artifacts ( $>2$ total), testing was repeated until the best quality study could be obtained. This study was used for analysis.

\section{Omnifield resolution perimetry}

ORP is United States Food and Drug Administration approved (Sinclair Technologies, Media, PA) for automated perimetry. ORP measures best-corrected $\log$ MAR visual acuity (BCVA) under mesopic conditions. In addition to measuring visual acuity at fixation, the BCVA is measured at various locations (17-24 intercepts) throughout the central $20^{\circ}$ of visual field. Landolt C's are presented on a monitor for $250 \mathrm{~ms}$ in one of four positions. Corrected for the $1 \mathrm{~m}$ testing distance, the patient signals their recognition of the correct " $\mathrm{C}$ " position by deflecting a joystick in the direction of the " $\mathrm{C}$ " opening on a response pad. Fixation is monitored automatically and an interactive algorithm adjusts the size of the Landolt C's to determine the threshold of the letter size below which the patient can no longer correctly respond, to arrive at the best corrected $\log$ MAR visual acuity for that intercept. The data are stored digitally and used to build a false-color map of mesopic BCVAs throughout the central $20^{\circ}$. Digital reporting includes the central acuity; the BA6 (the best visual acuity at any intercept within $6^{\circ}$ of fixation); the global macular acuity (GMA, or the average acuity from all intercepts weighted inversely from fixation), and the visual area (the area under the curve plotting thresholded BCVA versus intercept area), giving the area of various levels of measureable visual acuity. ORP was performed coincident with PERG testing.

\section{SDM treatment}

The treatment technique and laser parameters were identical in all patients. Following informed consent and pupillary dilation, topical proparacaine was applied to the cornea. A Mainster macular contact lens (Ocular Instruments, Mentor, Ohio, magnification factor $1.05 \times$ ) was placed on the cornea with the aid of viscoelastic. Under minimum slit-lamp illumination, the entire posterior retina, including the fovea, circumscribed by the major vascular arcades was "painted" with 1500-2000 confluent spot applications of SDM ("panmacular" treatment). The laser parameters used were $810 \mathrm{~nm}$ wavelength, 200 um aerial spot size, 5\% duty cycle; 1.4 Watt power, and $0.15 \mathrm{~s}$ duration (Oculight SLx, Iris Medical/Iridex Corp, Mountain View, California) [4]. Thus, each patient received identical treatment employing identical laser parameters. 


\section{Statistical analysis}

All the data were de-indentified prior to statistical analysis. Frequencies, means, and medians were calculated to summarize the data. Hierarchical linear models were performed to assess the association between the various measures before and after the treatment. The models included fixed eye effects and a random patient intercept to account for inter-eye correlation. Additional hierarchical linear models to explore the association between the difference (postminus pre-treatment) and pre-treatment values were also performed. Statistical analyses were performed using SAS 9.4 (SAS Institute; Cary, NC). Untreated fellow eyes of each patient served as controls.

\section{Results}

All patients presenting with RP since 2015 were offered and elected SDM treatment. All eyes were eligible and included for study, and none were excluded or lost to follow-up. This included 26 consecutive eyes of 15 patients, eight female and seven male, aged 16-69 years (avg. 47). (Table 1 in Supplemental Material)

\section{Retinal function by PERG}

No patient had significant cataracts or other media opacity that might interfere with PERG testing [5-12]. The PERG of all eyes was abnormal before, and after, SDM treatment [9]. PERG was improved by all measures following SDM treatment compared to fellow eye controls. Significant improvements (19/26 eyes, 73\%) were found in signal latency measures of the $24^{\circ}$ testing field. These included the direct latency measure, the $\operatorname{MagD}(\mu \mathrm{V})(p=0.0003)$; and the $\operatorname{MagD}(\mu \mathrm{V}) / \mathrm{Mag}(\mu \mathrm{V})$ ratio $(p<0.0001)$. (Table 2, supp) (Figs. 1 and 2a, b)

Linear regression analysis of the PERG indices showed significant negative correlations of all measures, indicating that the worse the pre-treatment values, the greater the post treatment improvements. (Table 3, supp)

\section{Visual acuity}

Best-corrected Snellen visual acuity was significantly improved following SDM compared to fellow eye controls, averaging $0.6 \log$ MAR units prior to treatment and 0.4 after $(p=0.02)$. (Table 1, supp)

\section{Omnifield resolution perimetry}

ORP testing was available on 23/26 eyes. In two eyes of one patient, ORP testing was within normal limits. Of the

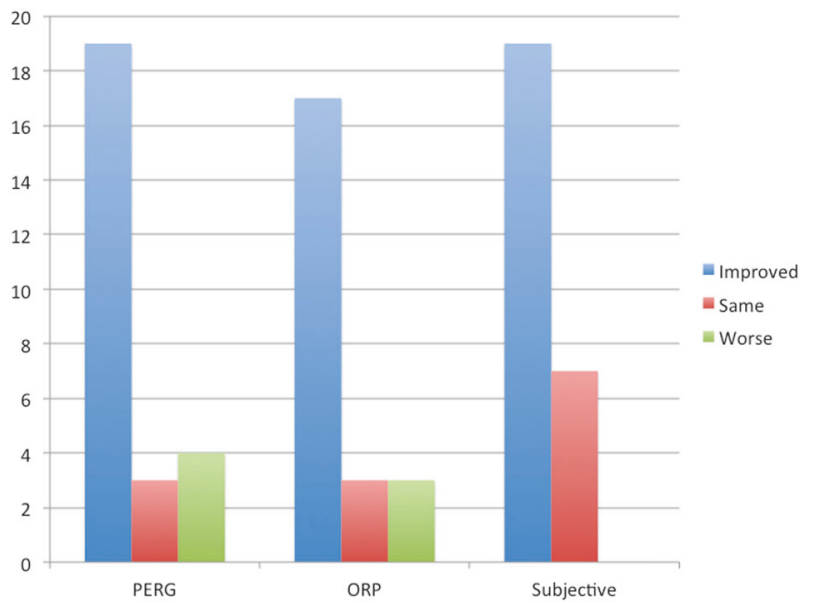

Fig. 1 Bar graph summarizing responses to SDM in RP by pattern electroretinography (PERG); omnifield resolution perimetry (ORP); and subjective patient experience

remaining 21 tested and improvable eyes, 3 eyes were worsened, 1 was unchanged, and $17(81 \%)$ eyes were improved by all measures compared to control eyes. Overall, the BA $6(p=0.02)$, GMA $(p=0.002)$, and visual area $(p=0.005)$ were significantly improved following SDM. (Table 4, supp) (Figs. 2b,c and 3).

Linear regression analysis of ORP data revealed significant associations in the negative direction of BA6 and visual area values, indicating that the worse the pretreatment values, the greater the post treatment improvements. (Table 5, supp)

\section{Subjective outcomes}

No patient reported subjective worsening after SDM. 19/26 eyes $(73 \%)$ were reported by patients to be subjectively improved after treatment. In all but one eye for which ORP testing was unavailable, subjective improvement was associated with improvement of the PERG and/or ORP. Subjective improvement was often noted within hours of treatment. The most common reports were of "brighter" vision; improved contrast; improved peripheral vision; and improved night vision, often marked by the renewed ability to see stars at night. Several reported improved color vision. Functionally, patients described increased ease of reading, navigating in low light, and less frequent stumbling over unseen objects. Dark adaptometry was not available to test these reports. Several patients reported that the improvements they noted following SDM treatment restored vision to levels not previously enjoyed for decade.

All patients reporting subjective improvement after treatment also reported loss or diminution the treatment effects, from 2 to 12 months postoperatively. In each case, repeat PERG and ORP testing confirmed a drop in retinal and visual function, compared to the first postop studies. In 
a

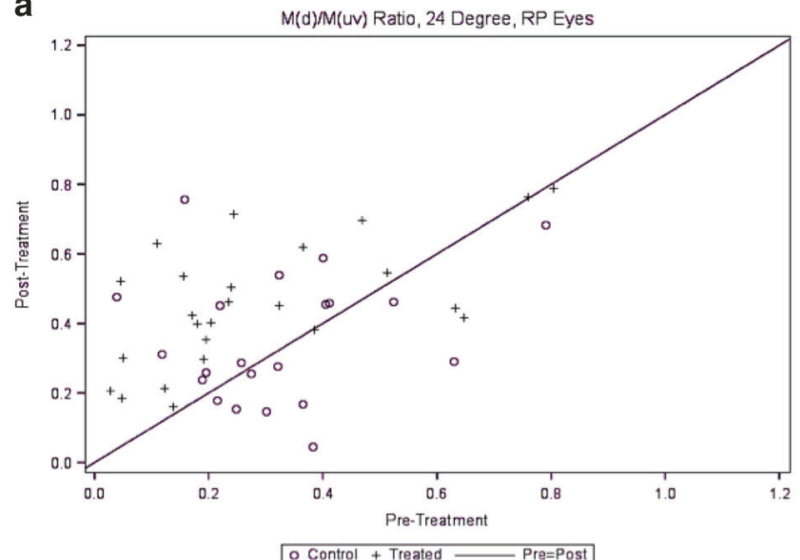

0 Control + Treated — Pre=Post

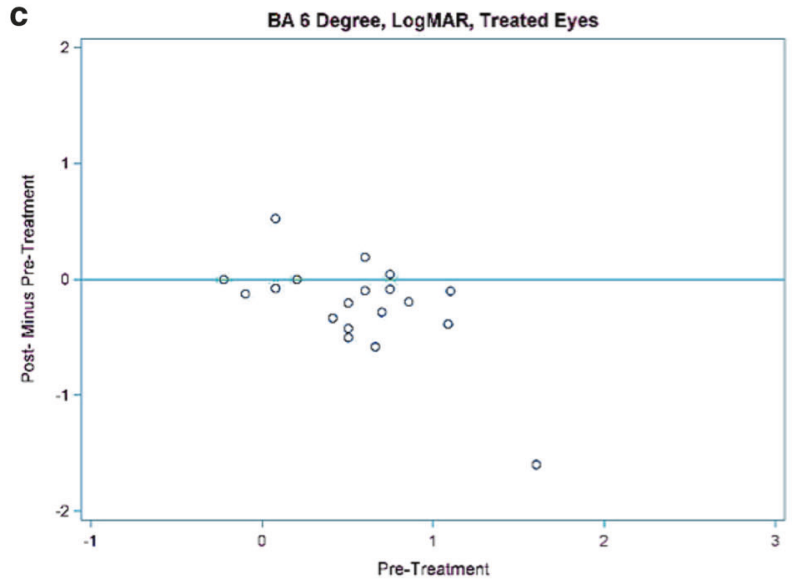

Fig. 2 a Scatter plot of pre vs. post treatment pattern electroretinography $(\mathrm{PERG}) \operatorname{MagD}(\mu \mathrm{V}) / \operatorname{Mag}(\mu \mathrm{V})$ ratios. $+=$ treated eye. $\mathrm{O}$ $=$ control eye. b Scatter plot of pre vs. post treatment $\operatorname{MagD}(\mu \mathrm{V})$ amplitudes. $+=$ treated eye. $0=$ control eye. c Plot of post-pre treatment ORP BA six values. Values below the line represent

each case, re-treatment restored both subjective and ORPmeasured visual improvements, as early as the following day. (Figs. 1, 3, and 4) (Table 6, supp)

\section{Discussion}

Eyes with RP may have measureable and potentially useful pattern electroretinography (PERG) responses despite severely subnormal to extinguished ERGs [3, 5-10]. While the ERG measures electrophysiologic activity by various methods (flash/Ganzfield, focal, and multifocal) in the photoreceptor layer, the PERG measures a steady-state response from the inner retina and ganglion cell layer to a continuous pattern reversal stimulus. Because PERG signals reflect input from mid- and outer retinal layers to the inner retina, the PERG has been used to evaluate RP $[8,9]$.

The PERG is generally abnormal in RP, reflecting disease severity. The PERG in RP may also be variable; in one b

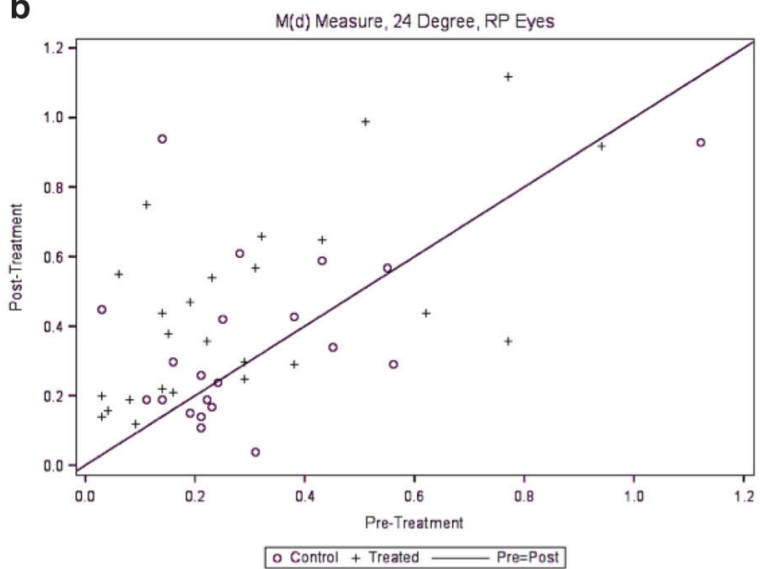

d

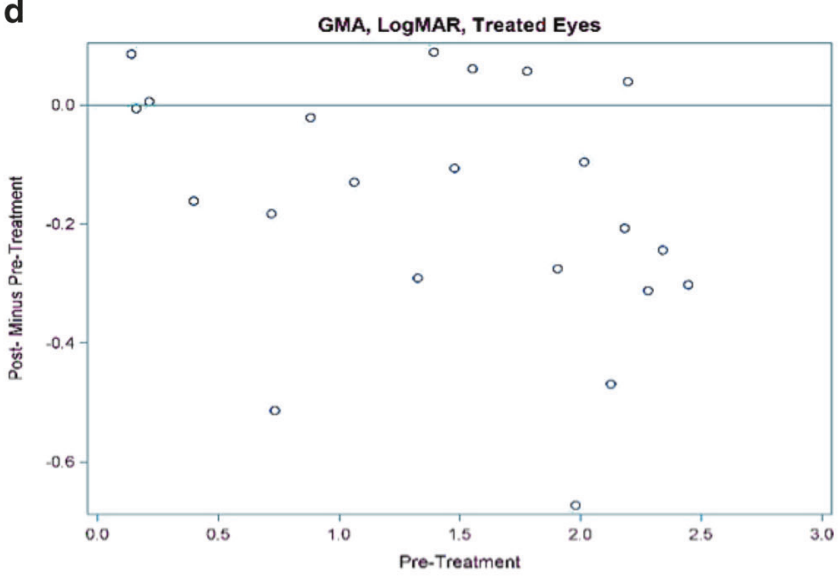

improvements after SDM treatment; above the line worsened after treatment. d Plot of post-pre treatment ORP GMA values. Values below the line represent improvements after SDM treatment; above the line worsened after treatment

study reproducible in only a third of eyes tested [9]. A number of factors may contribute to variable, and thus potentially unreliable, PERG results [5-12]. These include disease severity, media opacity, patient anxiety; and lack of uniformity in testing equipment, protocol, location and environment, and testing personnel [11]. To address intertest variability, averaging of several tests has been recommended [9]. As this is a report of outcomes from a clinical practice, serial testing was not a practical possibility, for a number of reasons. These include cost, time, and patient availability. However, testing was performed with single machine in a single location operated by a single-technician employing a single protocol with uniform methods. If a test result showed poor signal quality or excessive artifacts (two or more), testing was repeated to obtain a better quality study. A proprietary measure designed to adjust for intertest variability, the $\operatorname{MagD}(\mu \mathrm{V}) / \operatorname{Mag}(\mu \mathrm{V})$ ratio, was also used. Untreated fellow eyes served as controls, and statistical methods were employed to evaluate the results. Rather 
a

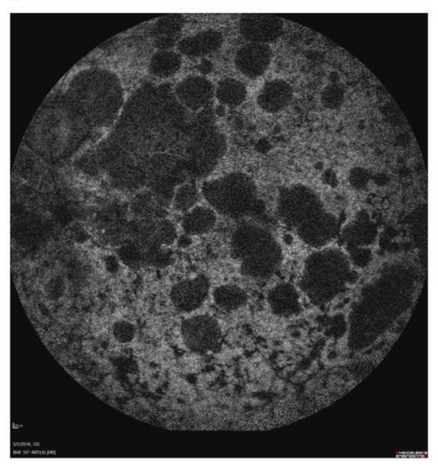

C

OS

Diagnosis \#1: No Diagnosis

Diagnosis \#2:

Diagnosis \#3:

Eye Notes:
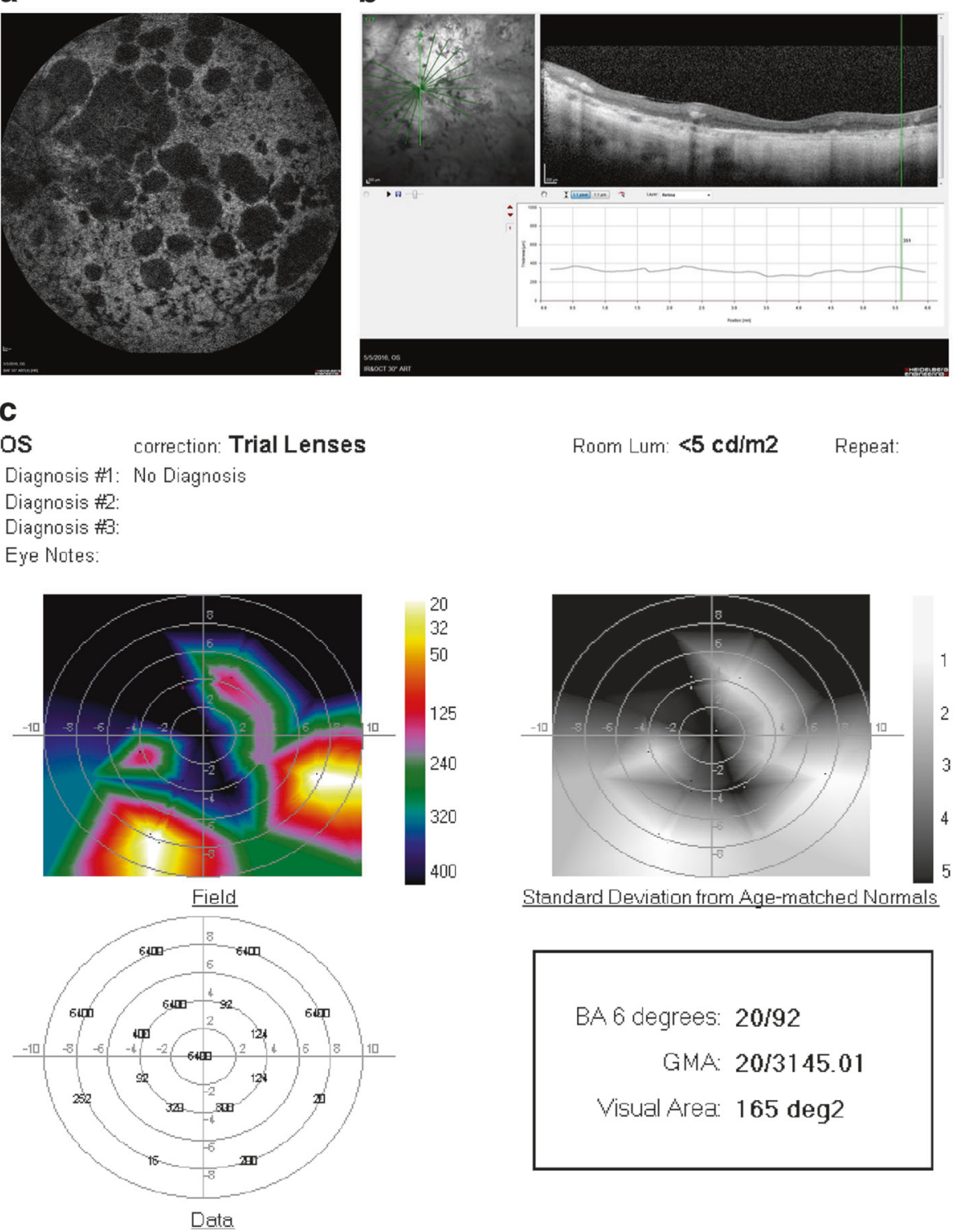

Room Lum: $<\mathbf{5}$ cdim2 Repeat:

correction: Trial Lenses

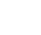

b

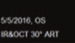


d

\section{os}

correction: Trial Lenses

Diagnosis H: No Diagnosis

Diagnosis \#2:

Diagnosis \#:

Eye Notes:
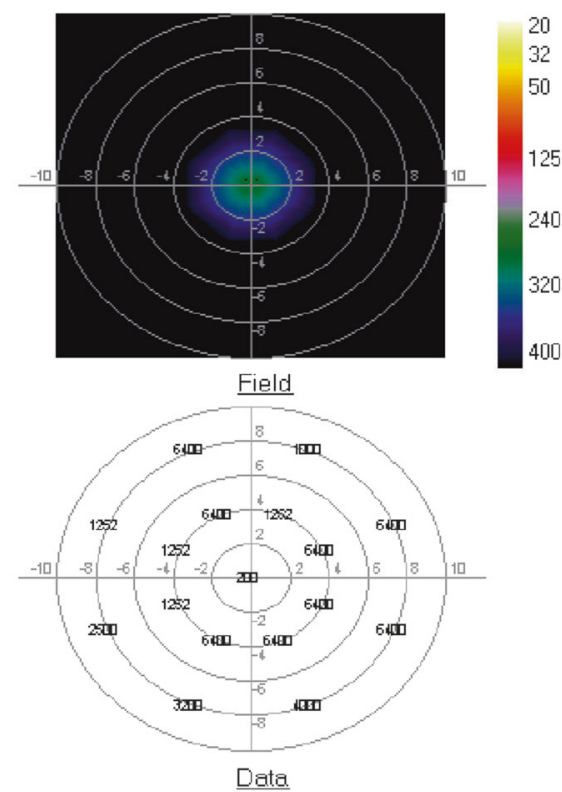

e

\section{os}

correction: Trial Lenses

Diagnosis H1: No Diagnosis

Diagnosis \#2:

Diagnosis \#:

Eye Notes:
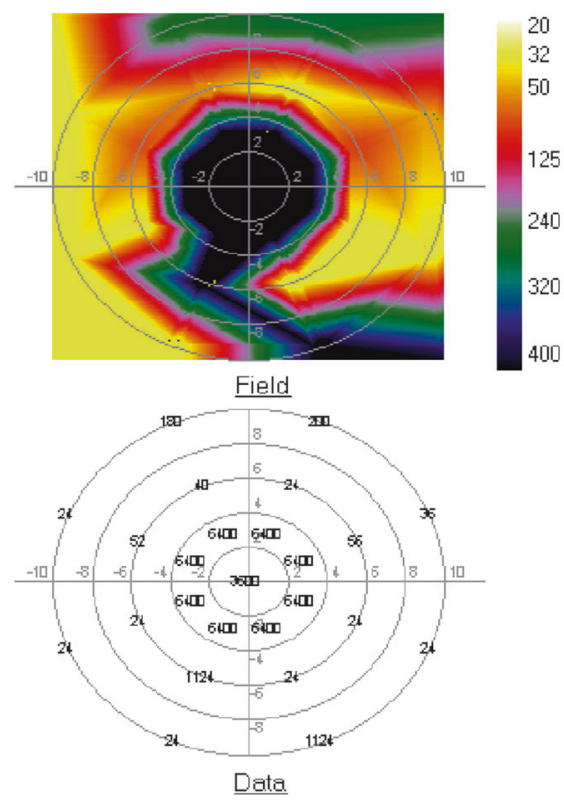

Fig. 3 a Fundus photograph of the left eye of a 51 year-old woman with RP and hand-motions visual acuity. b OCT showing severe diffuse retinal atrophy. Prior to SDM treatment the ORP of the left eye could not be recorded. c First recordable ORP, 1 month after SDM. Subjectively, the patient reported "brighter" vision and improved peripheral vision, able to see her feet and to see the color of her clothes for
Room Lum: $<5$ cdim2 Repeat:

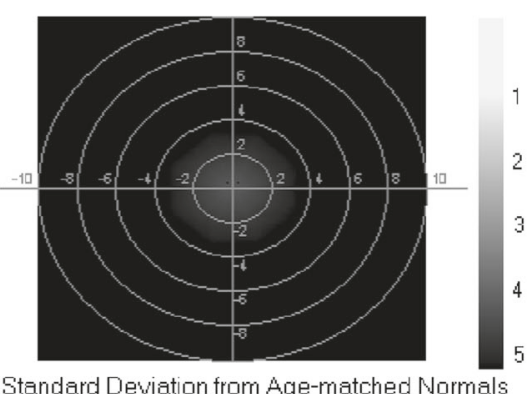

Standard Deviation from Age-matched Normals

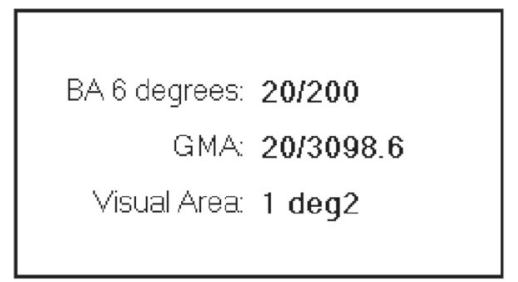

Room Lum: $<\mathbf{5}$ cdim2 Repeat:

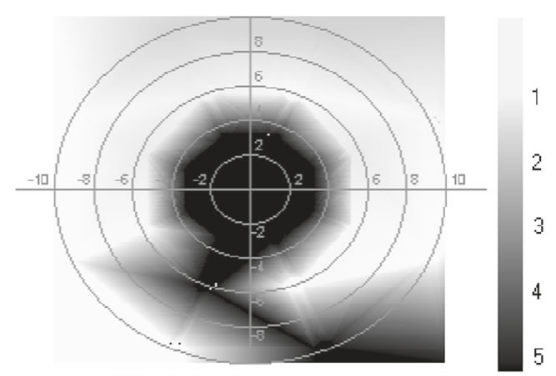

Standard Deviation from Age-matched Normals

BA 6 degrees: $20 / 24$

GMA: $20 / 3440.03$

Visual Area: $316 \mathrm{deg} 2$

the first time in years. $\mathbf{d}$ Three months after SDM. The patient reported gradual loss-of-treatment effect beginning 6-8 weeks after treatment. Re-treatment was performed. e ORP 2 weeks after repeat SDM. Subjectively, she reported recovery of visual function to the level noted after initial SDM treatment. BA6 best $\log$ MAR visual acuity within $6^{\circ}$ of fixation; GMA global macular $\log$ MAR visual acuity 
Fig. 4 Fifty-one-year-old man with RP initially treated by SDM RPT 2 years previously, without baseline perimetry. Visual acuity 20/200. After initial treatment the patient reported subjectively improved peripheral, night, and color vision. Fifteen months later the patient returned, reporting recurrent visual loss, in his left eye only, for 3-4 months. a ORP of the left eye that day demonstrating poor visual function. SDM was performed. One day following re-treatment, the ORP is improved by all measures. b Subjectively, the patient reported recovery of his prior level of visual function within a few hours of re-treatment. Chart visual acuity was unchanged at 20/200. BA6 best logMAR visual acuity within $6^{\circ}$ of fixation; GMA global macular $\log$ MAR visual acuity

\section{a}

os correction: Trial Lenses

Diagnosis \#1: No Diagnosis

Diagnosis 荆:

Diagnosis \#:

Eye Notes:
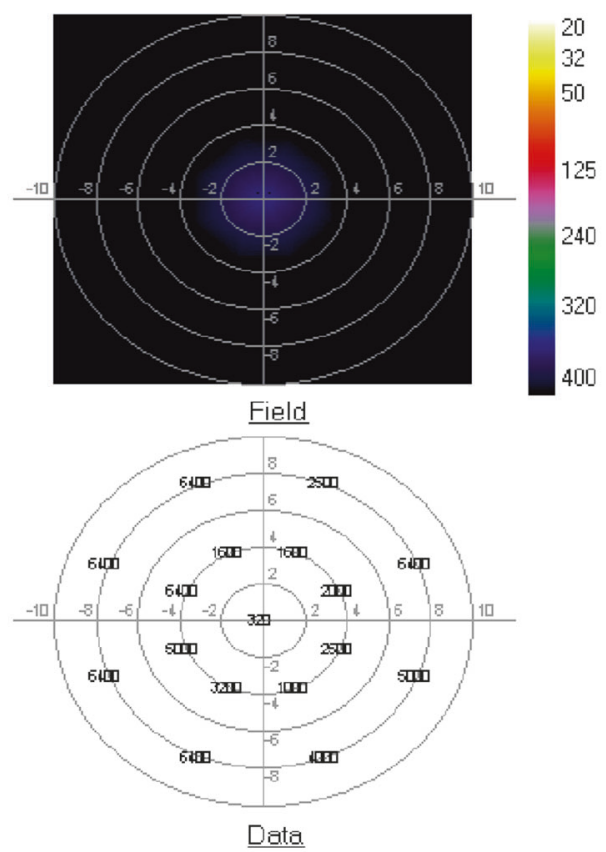

\section{b}

os

carrection: Trial Lenses

Diagnosis \#: No Diagnosis

Diagnosis ti2:

Diagnosis \#:

Eye Notes:

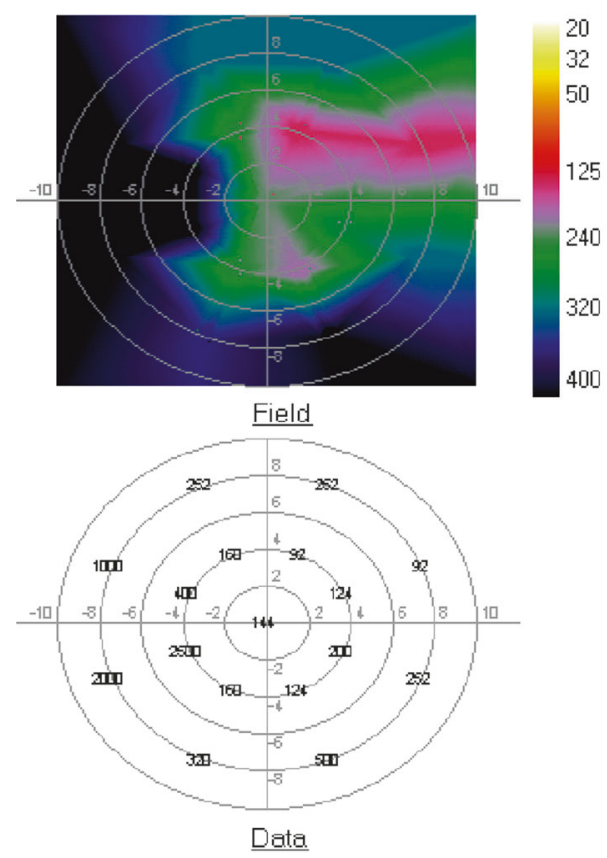

\section{Room Lum $<5$ cdim2 Repeat:}

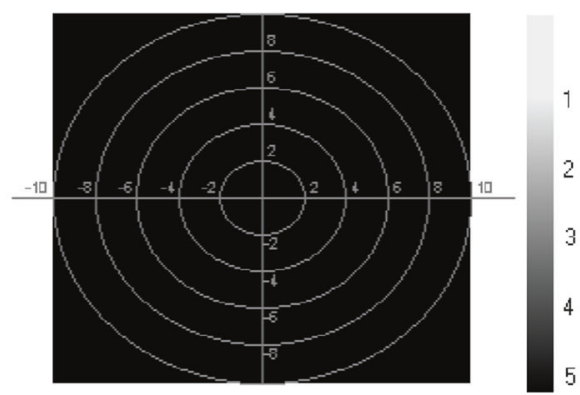

Standard Deviation from Age-matched Normals

BA, 6 degrees: $20 / 320$

GMA: $20 / 2813.07$

Visual Area: 0 deg2

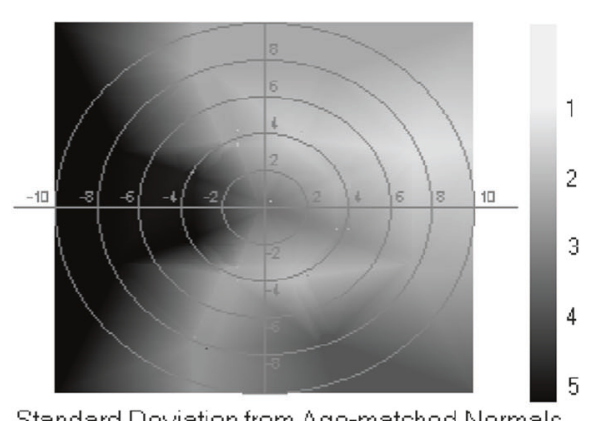

Standard Deviation from Age-matched Normals

BA 6 degrees: $20 / 92$

GMA: $20 / 405.47$

Visual Area: 119 deg2 
a

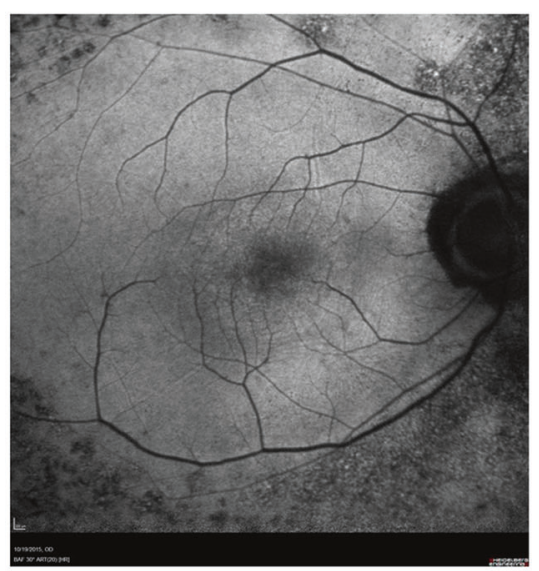

b

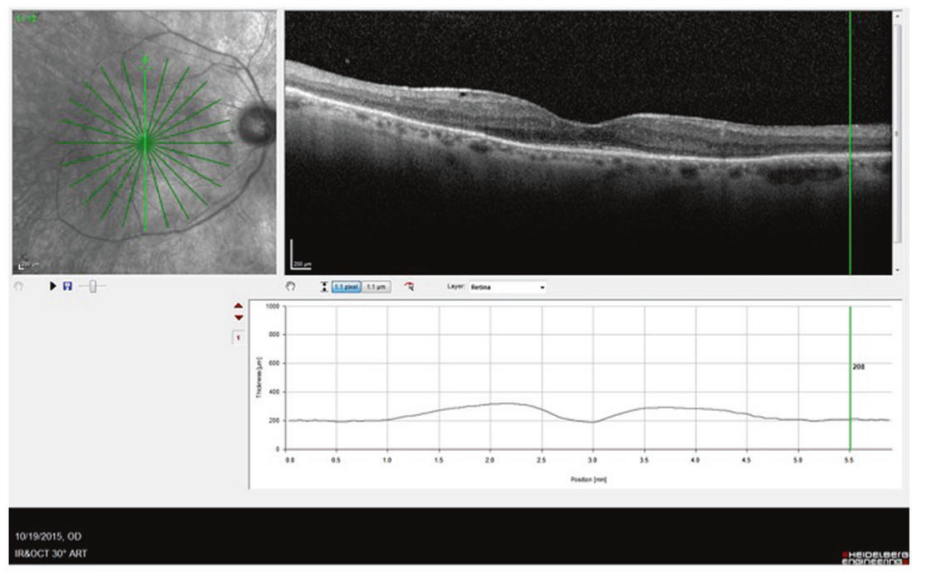

Room Lum: $<5$ cdim2 Repeat:

\section{$\mathrm{OD}$}

\section{Diagnosis \#1: No Diagnosis}

Diagnosis 2 :

Diagnosis \#3:

Eye Notes:
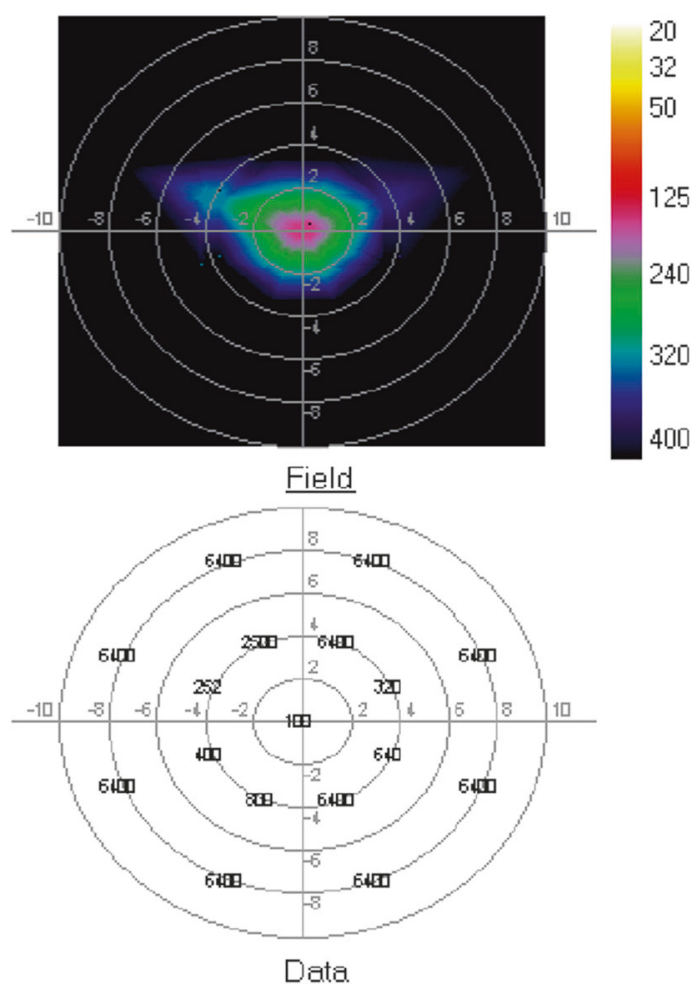

Fig. 5 For legend see next page

most normal prior to treatment. This "ceiling effect" leaves relatively less potential for improvement following treatment centrally; compared to the larger $24^{\circ}$ field which samples a larger area of retina that is also more dysfunctional, and thus has a greater capacity for improvement. In this study, and a prior study of dry AMD, linear regression

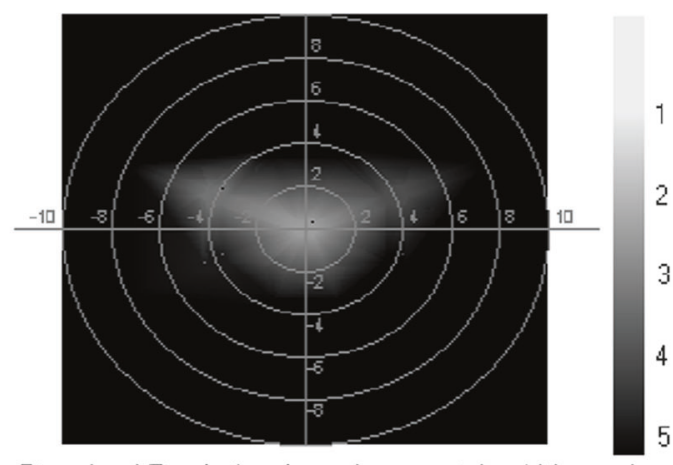

Standard Deviation from Age-matched Normals

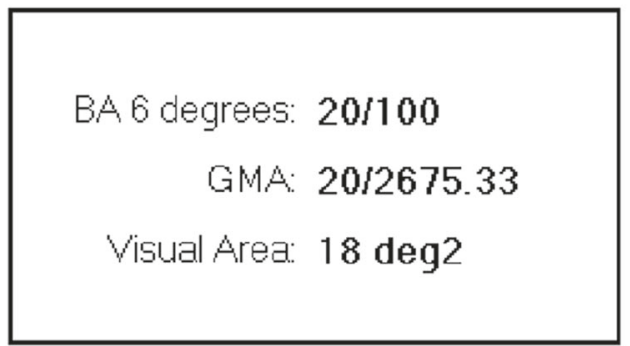

analysis reveals that the most dysfunctional eyes improve the most after SDM [4]. (Table 3, supp) In dry AMD, these are the eyes with the most extensive geographic atrophy [4]. It is important to understand that the improvements in PERG and ORP reported here and in dry AMD are not generally improvements from very bad to very good; rather, 


\section{d}

OD

Diagnosis \#: No Diagnosis

Diagnosis H2:

Diagnosis 瓶:

Eye Notes
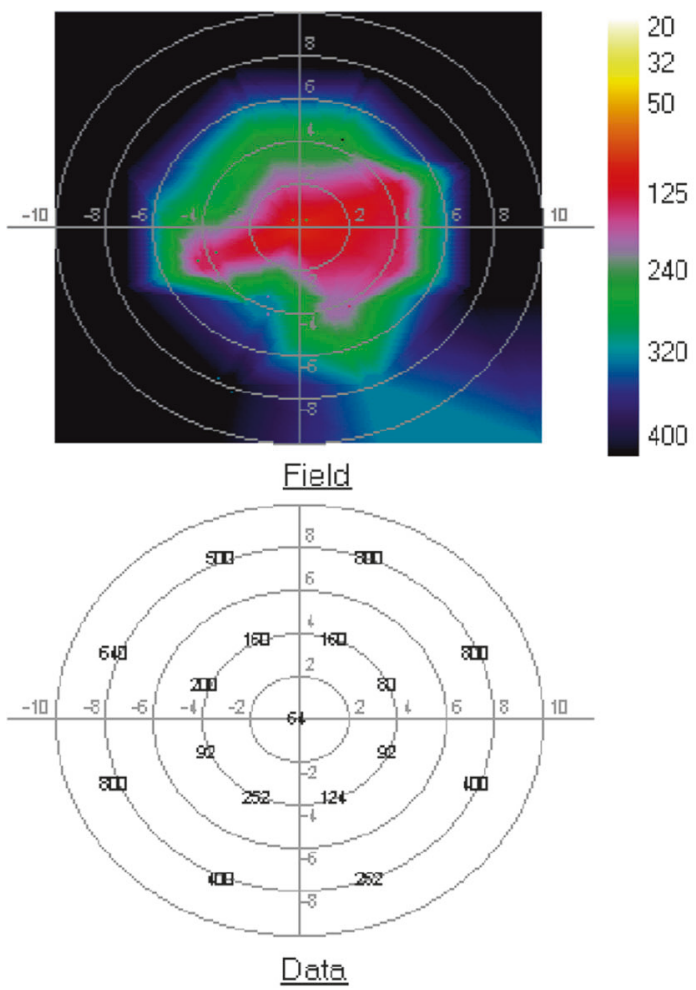

Fig. 5 a Fundus photograph of right eye 69-year-old man with RP, visual acuity 20/50. b OCT showing atrophy and loss of the RPE and photoreceptor layer outside the fovea. c ORP before and d 1 month after SDM. No improvement in all measures with enlargement of the

they are from very bad to not as bad. The results of OPR testing are illustrative. (Figs. 2b, c, 4, and 5) An effect of SDM is to restore function to non- or severely dysfunctional areas of the retina. This phenomenon can be seen clearly in the improvements in ORP visual area, the most improved measure. (Figs. 2b, c, 4, and 5) This reflects rescue of highly dysfunctional, but still viable, cells. Thus, as the sampling area of the $24^{\circ}$ stimulus field is over twice that of the $16^{\circ}$ field and encompasses more severely dysfunctional retina, the $24^{\circ}$ field improves more than the $16^{\circ}$ field in response to panmacular SDM treatment for RP.

ORP is a VA test. It uses correct identification of the position of a Landolt " $C$ " to obtain the best-corrected logMAR VA. Chart visual acuity may be insensitive to various types of visual dysfunction. Thus, ORP testing is done under mesopic conditions, improving both sensitivity to retinal disease and relevance to real-life visual requirements, including reading indoors [13-15]. In addition to fixation,

\section{Room Lum: $<5 \mathrm{~cd} / \mathrm{m} 2 \quad$ Repeat:}

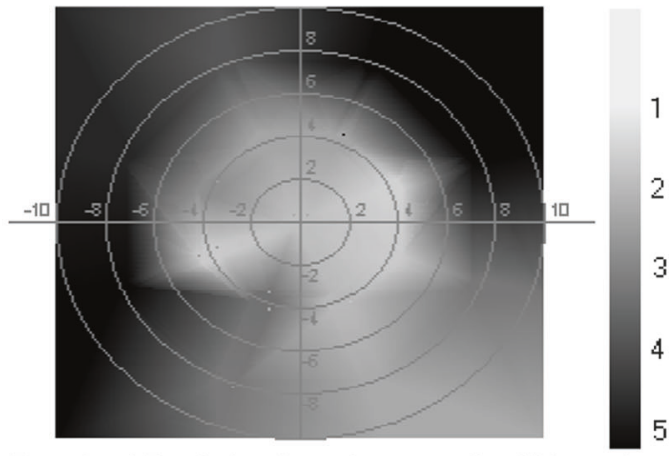

Standard Deviation from Age-matched Normals

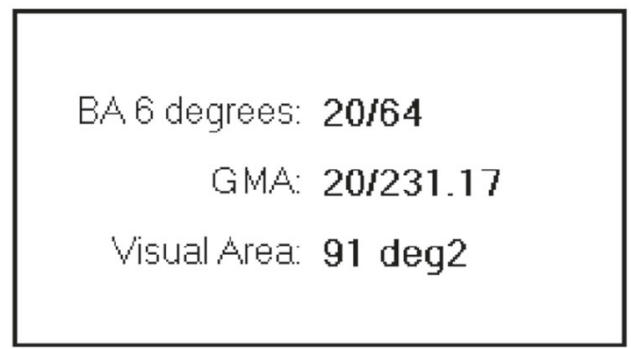

recordable visual area. Chart visual acuity was unchanged at 20/50. $B A 6$ best $\log$ MAR visual acuity within $6^{\circ}$ of fixation; GMA global macular $\log$ MAR visual acuity

ORP measures VA at various locations throughout the central $20^{\circ}$ of the visual field. Recorded data are then used to build a false-color map of VA throughout the central $20^{\circ}$ of the visual field ("resolution perimetry"). This facilitates topographic correlation of VA with retinal morphology. ORP is thus simple in conception and intuitive, rather than abstract, in interpretation. ORP has not been studied in comparison with traditional forms of automated perimetry, such as the Humphrey field analyzer (Carl Zeiss Meditech, Dublin, California). Thus, the results we report represent neither absolute values, nor results directly comparable to other testing methods. In this study, ORP was found to be sensitive to SDM treatment effects and clinically useful. (Figs. 2b, c-4) Comparisons of ORP results before and after treatment show consistent and significant improvements after treatment compared to control eyes; improvement in the central mesopic visual acuity (BA6) on the same order as improvements in chart acuities; and, paralleling PERG 
results, the greatest improvements in eyes with the worst preoperative indices by linear regression analysis. (Table 5, supp)

Because central visual function is best preserved in RP, the capacity for improvement is generally less in the fovea than from the more severely affected retina outside the fovea. Thus, ORP measures that include measures outside the fovea, where pre-treatment responses may be nonrecordable (the GMA and visual area), show the greatest magnitude of improvement after SDM treatment. (Figs. 45) All patients reporting subjective improvement after treatment also reported diminution or loss of the treatment effect as early as 6 weeks, and as late as 12 months after treatment, but usually within 2-3 months. These losses could be confirmed by ORP and PERG. (Fig. 3) Retreatment restored these losses, both subjectively, and by ORP and PERG testing. (Figs. 3, 4)

SDM employs a low-duty cycle micropulsed laser ("low intensity") applied over a wide area of confluent application ("high density") to achieve reliably safe (sublethal to the RPE) and effective treatment [16]. In almost 20 years of clinical use, SDM has been found to have only therapeutic effects. For conventional retinal laser applications, SDM is comparable to photocoagulation, but with better visual results [17-22]. In the absence of laser-induced retinal damage, SDM produces prompt improvements in retinal electrophysiology and visual function $[4,23]$. The effectiveness of SDM has lead to a new understanding of the mechanism of retinal laser treatment, described as a "reset to default" phenomenon, drawing an analogy to the reset function common to electronic devices [24]. Reset theory postulates that the principal effect of SDM is to address the commonalities of CPRs by activating retinal pigment epithelial (RPE) heat shock proteins (HSPs), leading to normalization of RPE and retina function, RPE cytokine expression, retinal autoregulation; decreasing chronic inflammation, and stimulating systemic immunomodulation [4, 25-42].

Each of these effects has been demonstrated by in vitro and in vivo studies of retinal laser treatment [29-32, 3537]. Acute inflammation is the most effective treatment for chronic inflammation [42]. In a recent study by Caballero, Kent and associates, SDM treatment in a murine model was found to elicit an acute inflammatory reaction in the absence of laser-induced tissue damage [37]. This same study documented recruitment of bone marrow-derived cells to the retina and RPE of both eyes, following SDM to just one eye [37]. These observations, as well as the findings of the current study, reflect the restorative "reset" processes initiated by sublethal laser treatment of the RPE [4, 24]. Such effects may, by improving retinal health and function, slow disease progression and thus be preventative with regard to the long-term risk of visual loss [25-43]. All patients in this study had flat ERGs, nyctalopia, clinical findings consistent with RP, and prior demonstration of concentric peripheral visual field loss by conventional automated perimetry. The degree of retinal and visual dysfunction varied according to disease severity. In one patient, ORP of both eyes (measuring the mesopic visual function within $10^{\circ}$ of fixation) was in the normal range prior to SDM. Treatment of such patients, with less advanced disease, reflects the primary precept of preventive treatment: to safely improve (retinal) function in hopes of slowing disease progression and the risk of functional (visual) loss [43]. Finally, reset theory also predicts that the effects of SDM in CPRs, such as RP will diminish with time; but that these effects should also be renewable and sustainable, without adverse effect, by periodic treatment $[4,24]$. The findings in this study support this notion. However, further investigation and confirmation by subsequent study is required. (Figs. 3 and 4 )

This report is limited by retrospective data collection, small sample size, short follow-up, and the experience of a single-surgeon employing a novel treatment. However, the results are controlled, consistent, and robust. Significant improvements in retinal function measured by PERG, and visual acuity and function measured by ORP were observed in RP following panmacular SDM [4, 24]. These findings are consistent with all prior studies of SDM [4, 25-37]. In the absence of other effective therapies, the safety, efficacy, low cost, and simplicity of SDM suggest it may be useful in the treatment RP. Improved retinal and visual function, such as observed in this study, have been suggested as a reliable surrogate indicators of slowed disease progression [38]. If confirmed by larger randomized prospective studies, the findings of this study may lead to new areas of study and therapy not previously considered for RP, and other chronic progressive retinopathies. Further study is warranted.

\section{Summary}

\section{What was known before}

- To date, there is no non-specific treatment of any benefit to patients with retinitis pigmentosa.

- Functional improvement is a reliable surrogate for prevention and/or slowed disease progression.

- Retinal laser treatment has no role in the treatment of retinitis pigmentosa.

- No treatment improves electrophysiology in RP.

- No treatment improves visual fields in RP.

- No treatment improves visual acuity in RP.

\section{What this study adds}

- SDM appears to improve RP independent of underlying genomics.

- The improvements in retinal and visual function in RP 
produced by SDM may slow progression and reduce visual loss.

- The unique characteristics of SDM may create a role for SDM in the treatment of RP.

- SDM improves retinal function by electrophysiology in RP. This suggests that the promise of new RP treatments should be evaluated by confirmation of improved retinal electrophysiology function

- SDM improves mesopic visual fields in RP.

- SDM improves mesopic and photopic VA in RP.

Acknowledgements The author would like to thank Taylor Blachley, MS, for his assistance in statistical data analysis. Ojai Retinal Technologies, LLC, Ojai, California: Managing member, patent, equity. Retinal Protection Sciences, LLC, Ojai, California: CEO, CMO, equity.Ocular Proteomics, LLC, Towson, Maryland: Consultant. Replenish, Inc, Pasadena, California: Patent, equity.

\section{Compliance with ethical standards}

Conflict of interest The author declares that they have no competing interests.

Open Access This article is licensed under a Creative Commons Attribution-NonCommercial-NoDerivatives 4.0 International License, which permits any non-commercial use, sharing, distribution and reproduction in any medium or format, as long as you give appropriate credit to the original author(s) and the source, and provide a link to the Creative Commons license. You do not have permission under this license to share adapted material derived from this article or parts of it. The images or other third party material in this article are included in the article's Creative Commons license, unless indicated otherwise in a credit line to the material. If material is not included in the article's Creative Commons license and your intended use is not permitted by statutory regulation or exceeds the permitted use, you will need to obtain permission directly from the copyright holder. To view a copy of this license, visit http://creativecommons.org/licenses/by-nc-nd/4.0/.

\section{References}

1. Bourne RR, Stevens GA, White RA. et al.Vision Loss Expert Group Causes of vision loss worldwide, 1990-2010: a systematic analysis. Lancet Glob Health. 2013;1:339-49.

2. Kastner S, Thiemann IJ, Dekomien G, Petrasch-Parwez E, et al. Exome sequencing reveals AGBL5 as novel candidate gene and additional variants for retinitis pigmentosa in five Turkish families. Invest Ophthalmol Vis Sci. 2015;56:8045-53.

3. Sacchetti M, Mantelli F, Merlo D, Lambiase A. Systematic review of randomized clinical trials on safety and efficacy of pharmacological and nonpharmacological treatments for retinitis pigmentosa. J Ophthalmol. 2015;2015:737053.

4. Luttrull JK, Margolis BWL. Functionally guided retinal protective therapy as prophylaxis for age-related and inherited retinal degenerations. A pilot study. Invest Ophthalmol Vis Sci. 2016;57:265-75

5. McCulloch DL, Marmor MF, Brigell MG, et al. ISCEV Standard for full-field clinical electroretinography (2015 update). Doc Ophthalmol. 2015;130:1-12.

6. Porciatti V, Ventura LM. Normative data for a user-friendly paradigm for pattern electroretinogram recording. Ophthalmology. 2004;111:161-8.
7. Riggs LA. Electroretinography. Vis Res. 1986;26:1443-59.

8. Robson AG, Michalides M, Saiban Z, et al. Functional characteristic of patients with retinal dystrophy that manifest abnormal parafoveal annuli of high density fundus autofluorescence; a review and update. Doc Ophthalmol. 2008;116:79-89.

9. Janaky M, Palffy A, Horvath G, et al. Pattern-reversal electroretinograms and visual evoked potentials in retinitis pigmentosa. Doc Ophthalmol. 2008;117:27-36.

10. Arden GB, Vaegan HoggCR. Clinical and experimental evidence that the pattern electroretinogram (PERG) is generated in more proximal retinal layers than the focal electroretinogram (FERG). Ann N Y Acad Sci. 1982;388:580-607.

11. Odom JV, Holder GE, Feghali JG, Cavender S. Pattern electroretinogram intrasession reliability: a two center comparison. Clin Vis Sci. 1992;7:263-81

12. Bowd C, Trafreshi A, Zangwill LA, et al. Repeatability of pattern electrogram measurements using a new paradigm optimized for glaucoma detection. J Glaucoma. 2009;18:437-42.

13. Sebag J, Sadun AA, Pierce EA. for the Council of The American Ophthalmological Society Paradigm Shifts in Ophthalmic Diagnostics. Trans Am Ophthalmol Soc. 2016;114:WP1

14. Barteselli G, Gomez ML, Doede AL, et al. Visual function assessment in simulated real-life situations in patients with agerelated macular degeneration compared to normal subjects. Eye. 2014;28:1231-8.

15. Owsley C, Clark ME, Huisingh CE, et al. Visual function in older eyes in normal macular health: association with incident early agerelated macular degeneration 3 years later. Invest Ophthalmol Vis Sci. 2016;57:1782-9.

16. Luttrull JK, Musch MC, Mainster MA. Subthreshold diode micropulse photocoagulation for the treatment of clinically significant diabetic macular edema. $\mathrm{Br} \mathrm{J}$ Ophthalmol. 2005;89:74-80.

17. Luttrull JK, Dorin G. Subthreshold diode micropulse photocoagulation as invisible retinal phototherapy for diabetic macular edema. A review. Curr Diabetes Rev. 2012;8:274-84.

18. Chen G, Tzekov R, Li W, Jiang F, et al. Subthreshold micropulse diode laser versus conventional laser photocoagulation for diabetic macular edema: a meta-analysis of randomized controlled trials. Retina. 2016;36:2059.

19. Brader HS, Young LH. Subthreshold diode micropulse laser: a review. Semin Ophthalmol. 2016;31:30-9.

20. Luttrull JK, Sramek C, Palanker D, et al. Long-term safety, highresolution imaging, and tissue temperature modeling of subvisible diode micropulse photocoagulation for retinovascular macular edema. Retina. 2012;32:375-86.

21. Lavinsky D, Cardillo JA, Melo LA Jr., et al. Randomized clinical trial evaluating mETDRS versus normal or high-density micropulsephotocoagulation for diabetic macular edema. Invest Ophthalmol Vis Sci. 2011;52:4314-23.

22. Luttrull JK, Sinclair SH. Safety of transfoveal subthreshold diode micropulse laser (SDM) for fovea-involving diabetic macular edema in eyes with good visual acuity. Retina. 2014;34:2010-20.

23. Vujosevic S, Bottega E, Casciano M, et al. Microperimetry and fundus autofluorescence in diabetic macular edema. Subthreshold micropulse diode laser versus modified Early Treatment Diabetic Retinopathy Study Laser photocoagulation. Retina. 2010;30:908-16.

24. Luttrull JK, Chang DB, Margolis BWL, Dorin G, Luttrull DK. Laser re-sensitization of medically unresponsive neovascular agerelated macular degeneration: efficacy and implications. Retina. 2015;35:1184-94.

25. Gao X, Xing D. Molecular mechanisms of cell proliferation induced by low power laser irradiation. J Biomed Sci. 2009;16:4.

26. Karu TI, Kolyakov SF. Exact action spectra for cellular responses relevant to phototherapy. Photomed Laser Surg. 2005;23:355-61. 
27. Beckham JT. The role of heat shock protein 70 in laser irradiation and thermal preconditioning. $\mathrm{PhD}$ dissertation, Vanderbilt University Press (2008). Nashville, Tennessee.

28. Robert RL. Energetics of protein folding. J Mol Biol. 2007;371:283-301.

29. Iwami H, Pruessner J, Shariaki K, et al. Protective effect of a laserinduced sub-lethal temperature rise on RPE cells from oxidative stress. Exp Eye Res. 2014;124:37-47.

30. Hattenbach LO, Beck KF, Pfeilschifter J, et al. Pigmentepithelium- derived factor is upregulated in photocoagulated human retinal pigment epithelial cells. Ophthalmic Res. 2005;37:341-6.

31. Basso FG, Pansani TN, Soares DG, et al. Biomodulation of inflammatory cytokines related to oral mucositis by low-level laser therapy. Photochem Photobiol. 2015;91:952-6.

32. Flaxel C, Bradle J, Acott T, Samples JR. Retinal pigment epithelium produces matrix metalloproteinases after laser treatment. Retina. 2007;27:629-34.

33. Kolomeyer AM, Zarbin MA. Trophic factors in the pathogenesis and therapy for retinal degenerative diseases. Surv Ophthalmol. 2014;59:134-65.

34. Kregel K. Heat shock proteins: modifying factors in physiological stress responses and acquired thermotolerance. J Appl Physiol. 2002;5:2177-86.

35. Sramek C, Mackanos M, Spitler R, et al. Non-damaging retinal phototherapy: dynamic range of heat shock protein expression. Invest Ophthalmol Vis Sci. 2011;52:1780-7.
36. Inagaki K, Shuo T, Katakura K, et al. Sublethal photothermal stimulation with a micropulse laser induces heat shock protein expression in ARPE-19 cells. J Ophthalmol. 2015;2015:729792.

37. Caballero SKD, Nilanjana Sengupta N. Bone marrow-derived cell recruitment to the neurosensory retina and retinal pigment epithelial cell layer following subthreshold retinal phototherapy. Invest Ophthalmol Vis Sci. 2017;58:5164-76.

38. Hageman GS, Anderson DH, Johnson LV, et al. A common haplotype in the complement regulatory gene factor $\mathrm{H}(\mathrm{HF} 1 / \mathrm{CFH})$ predisposes individuals to age-related macular degeneration. Proc Natl Acad Sci USA. 2005;102:7227-32.

39. Frydman J. Folding of newlytranslated proteins in vivo: the role of molecular chaperones. Annu Rev Biochem. 2001;70:603-47.

40. Lanneau D, de Thonel A, Maurel S, Didelot C, Garrido C. Apoptosis versus cell differentiation: role of heat shock proteins HSP90, HSP70 and HSP27. Prion. 2007;1:53-60.

41. Franceschi $\mathrm{C}$, Bonafe $\mathrm{M}$, Valensin $\mathrm{S}$, et al. Inflamm-aging. An evolutionary perspective on immunosenescence. Ann N Y Acad Sci. 2000;908:244-54.

42. Medzhitov R. Origin and physiological roles of inflammation. Nature. 2008;454:428-35.

43. Scott IU, Jackson GR, Quillen DA, et al. Effect of doxycycline vs placebo on retinal function and diabetic retinopathy progression in patients with severe nonproliferative or non-high-risk proliferative diabetic retinopathy: a randomized clinical trial. JAMA Ophthalmol. 2014;132:535-43. 